\title{
TOTALLY ACYCLIC COMPLEXES AND LOCALLY GORENSTEIN RINGS
}

\author{
LARS WINTHER CHRISTENSEN AND KIRIKO KATO
}

\begin{abstract}
A commutative noetherian ring with a dualizing complex is Gorenstein if and only if every acyclic complex of injective modules is totally acyclic. We extend this characterization, which is due to Iyengar and Krause, to arbitrary commutative noetherian rings, i.e. we remove the assumption about a dualizing complex. In this context Gorenstein, of course, means locally Gorenstein at every prime.
\end{abstract}

\section{The Theorem}

Let $R$ be a commutative ring. A complex $A$ of $R$-modules with $\mathrm{H}(A)=0$ is called acyclic. An acyclic complex $P$ of projective $R$-modules is called totally acyclic if $\operatorname{Hom}_{R}(P, Q)$ is acyclic for every projective $R$-module $Q$; likewise an acyclic complex $I$ of injective $R$-modules is called totally acyclic if $\operatorname{Hom}_{R}(E, I)$ is acyclic for every injective $R$-module $E$. Finally, an acyclic complex $F$ of flat $R$-modules is called $F$-totally acyclic if $F \otimes_{R} E$ is acyclic for every injective $R$-module $E$.

The invariant splf $R=\sup \left\{\right.$ proj. $\operatorname{dim}_{R} F \mid F$ is a flat $R$-module $\}$ is finite if and only if every flat $R$-module has finite projective dimension. Indeed, a direct sum of flat modules is flat with proj. $\operatorname{dim}_{R}\left(\bigoplus_{\lambda \in \Lambda} F_{\lambda}\right)=\sup _{\lambda \in \Lambda}\left\{\right.$ proj. $\left.\operatorname{dim}_{R} F_{\lambda}\right\}$. A ring with splf $R \leq n$ is called $n$-perfect; in particular, a 0 -perfect ring is a perfect ring in the sense of Bass' Theorem P. With these definitions in place we can state our:

Theorem 1.1. Let $R$ be a commutative noetherian ring. Among the conditions

(i) The local ring $R_{\mathfrak{p}}$ is Gorenstein for every prime ideal $\mathfrak{p}$ of $R$

(ii) Every acyclic complex of injective $R$-modules is totally acyclic

(iii) Every acyclic complex of flat $R$-modules is F-totally acyclic

(iv) Every acyclic complex of projective R-modules is totally acyclic the following implications hold

$$
(i) \Longleftrightarrow(i i) \Longleftrightarrow(i i i) \Longrightarrow(i v) \text {. }
$$

Moreover, if splf $R$ is finite, then all four conditions are equivalent.

The proof is given in the next section. Here we continue with a discussion of the precursors of the theorem and the condition splf $R<\infty$; in the rest of this section $R$ is assumed to be noetherian.

Date: 10 February 2017.

2010 Mathematics Subject Classification. 13D02; 13H10.

Key words and phrases. Gorenstein ring, totally acyclic complex.

The work was done while L.W.C. visited the Department of Mathematics and Information Sciences at Osaka Prefecture University. The visit took place under the Visitor Program of the Graduate School of Science, and the support and hospitality is acknowledged with gratitude. 
If the ring $R$ has finite Krull dimension - if it is local, in particular - then it is Gorenstein if and only if $\operatorname{inj} . \operatorname{dim}_{R} R$ is finite. It is standard to call a ring that satisfies part $(i)$ in the theorem Gorenstein, but notice that such a ring need not have finite injective dimension as a module over itself. Nagata's regular ring of infinite Krull dimension serves as an example.

Equivalence of the conditions $(i),(i i)$, and $(i v)$ in the theorem was proved by Iyengar and Krause [6, Corollary 5.5] under the assumption that $R$ has a dualizing complex. That assumption implies that $R$ has finite Krull dimension; see Hartshorne [5. Corollary V.7.2], and as we recall next that implies splf $R<\infty$.

For a ring of finite Krull dimension $d$, one has splf $R \leq d$ by work of Gruson and Raynaud [10, Theorem II.(3.2.6)] and Jensen [7, Proposition 6]. In this context the equivalence of conditions $(i),(i i i)$, and $(i v)$ in the theorem was proved by Murfet and Salarian [8, Theorem $4.27 \&$ Corollary 4.28], and the equivalence of all four conditions was recently proved by Estrada, $\mathrm{Fu}$, and Iacob $[3$.

Thus, the novelty of Theorem 1.1 is twofold; it establishes:

- the equivalence of conditions $(i)$ - $($ iii $)$ for rings of infinite Krull dimension.

- the equivalence of $(i)-(i v)$ for certain rings of infinite Krull dimension. Indeed, by a result of Gruson and Jensen [4, Theorem 7.10] one has splf $R \leq n+1$ if $R$ has cardinality at most $\aleph_{n}$ for some natural number $n$.

\section{The PROOF}

Our argument for $(i i) \Rightarrow(i)$ is an application of a result due to Štovíček [1]. The rest of the proof is based on, mostly, standard arguments that can be found in some form in the literature. We repeat them for the reader's convenience and to clarify under which conditions they apply.

Outline. For any commutative ring $R$, one can consider the following conditions:

(1) Every acyclic complex of projective $R$-modules is F-totally acyclic.

(2) Every acyclic complex of injective $R$-modules is totally acyclic.

(3) Every acyclic complex of flat $R$-modules is F-totally acyclic.

(4) Every acyclic complex of projective $R$-modules is totally acyclic.

For a noetherian ring $R$ one can also consider:

( $3^{\text {bis }}$ ) The local ring $R_{\mathfrak{p}}$ is Gorenstein for every prime ideal $\mathfrak{p}$ of $R$.

The proof of Theorem 1.1 goes as follows. These implications always hold:

$$
(1) \Longleftrightarrow(3) \Longleftarrow(2) \text { see Claims } 2.2 \text { and } 2.3 \text {. }
$$

Under the assumption that $R$ is coherent, one has

$$
(1) \Longrightarrow(4) \quad \text { see [8, Lemma 4.20] or Claim 2.4, }
$$

and if $R$ is coherent with splf $R$ finite, then one also has

$$
(1) \Longleftarrow(4) \text { see [8, Lemma 4.20] or Claim 2.5. }
$$

If $R$ is noetherian, then one has

$$
\left(3^{\text {bis }}\right) \Longleftrightarrow(3) \quad \text { see [8, Theorem 4.27] or Claim 2.6] }
$$

and

$$
\left(3^{\text {bis }}\right) \Longrightarrow(2) \quad \text { see Claim } 2.1 \text {. }
$$

This finishes the proof of Theorem 1.1

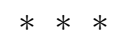


Recall that an $R$-module $C$ is called cotorsion if $\operatorname{Ext}_{R}^{1}(F, C)=0$ holds for every flat $R$-module $F$.

Claim 2.1. Let $R$ be noetherian. If the local ring $R_{\mathfrak{p}}$ is Gorenstein for every prime ideal $\mathfrak{p}$ in $R$, then every acyclic complex of injective $R$-modules is totally acyclic.

Proof. Let $I$ be an acyclic complex of injective $R$-modules. It is sufficient to show that $\operatorname{Hom}_{R}(E, I)$ is acyclic for every indecomposable injective $R$-module $E=E(R / \mathfrak{p})$. Fix such a module, it has an $R_{\mathfrak{p}}$-module structure, and the $R$-action factors through this structure, so standard adjunction yields

$$
\operatorname{Hom}_{R}(E, I) \cong \operatorname{Hom}_{R}\left(R_{\mathfrak{p}} \otimes_{R_{\mathfrak{p}}} E, I\right) \cong \operatorname{Hom}_{R_{\mathfrak{p}}}\left(E, \operatorname{Hom}_{R}\left(R_{\mathfrak{p}}, I\right)\right) .
$$

By [11, Corollary 5.9] the cycle submodules of the complex $I$ are cotorsion, so the complex $\operatorname{Hom}_{R}\left(R_{\mathfrak{p}}, I\right)$ of injective $R_{\mathfrak{p}}$-modules is acyclic. As $R_{\mathfrak{p}}$ is Gorenstein, the injective module $E$ has finite projective dimension, and it follows that $\operatorname{Hom}_{R_{\mathfrak{p}}}\left(E, \operatorname{Hom}_{R}\left(R_{\mathfrak{p}}, I\right)\right)$ and hence $\operatorname{Hom}_{R}(E, I)$ acyclic.

Claim 2.2. Every acyclic complex of projective R-modules is F-totally acyclic if and only if every acyclic complex of flat R-modules is F-totally acyclic.

Proof. The "if" part is trivial as projective modules are flat. For the converse, let $F$ be an acyclic complex of flat modules. It follows from [9, Propositions 8.1 $\&$ 9.1] that, in the homotopy category of complexes of flat modules, there is a distinguished triangle $P \rightarrow F \rightarrow C \rightarrow$, where $P$ is a complex of projective modules and $C$ is pure acyclic; i.e. $\mathrm{H}(C)=0=\mathrm{H}\left(C \otimes_{R} M\right)$ for every module $M$. It follows that $P$ is acyclic, so by assumption it is F-totally acyclic, and hence so is $F$.

Claim 2.3. If every acyclic complex of injective $R$-modules is totally acyclic, then every acyclic complex of flat $R$-modules is F-totally acyclic.

Proof. Let $F$ be an acyclic complex of flat $R$-modules. The character complex $\operatorname{Hom}_{\mathbb{Z}}(F, \mathbb{Q} / \mathbb{Z})$ is an acyclic complex of injective $R$-modules, so the complex

$$
\operatorname{Hom}_{R}\left(E, \operatorname{Hom}_{\mathbb{Z}}(F, \mathbb{Q} / \mathbb{Z})\right) \cong \operatorname{Hom}_{\mathbb{Z}}\left(F \otimes_{R} E, \mathbb{Q} / \mathbb{Z}\right)
$$

is by assumption acyclic for every injective $R$-module $E$. It follows that $F \otimes_{R} E$ is acyclic for every injective $R$-module $E$.

The classes of flat modules and cotorsion modules make up a complete cotorsion pair; that is a theorem of Bican, El Bashir, and Enochs [1]. In particular, there is for every $R$-module $M$ an exact sequence $0 \rightarrow M \rightarrow C \rightarrow F \rightarrow 0$ where $C$ is cotorsion and $F$ is flat. If $M$ is flat, then so is $C$, so by iteration a flat $R$-module admits a, possibly infinite, coresolution by flat cotorsion modules.

Claim 2.4. Let $R$ be coherent and $P$ be an acyclic complex of projective $R$-modules. If $P$ is $F$-totally acyclic, then $\operatorname{Hom}_{R}(P, G)$ is acyclic for every flat $R$-module $G$; in particular, $P$ is totally acyclic.

Proof. For every injective $R$-module $E$ the complex $P \otimes_{R} E$ and hence

$$
\operatorname{Hom}_{\mathbb{Z}}\left(P \otimes_{R} E, \mathbb{Q} / \mathbb{Z}\right) \cong \operatorname{Hom}_{R}\left(P, \operatorname{Hom}_{\mathbb{Z}}(E, \mathbb{Q} / \mathbb{Z})\right)
$$

is acyclic. By [12, Lemma 3.2.3] an $R$-module is cotorsion and flat if and only if it is a direct summand of the character module $\operatorname{Hom}_{\mathbb{Z}}(E, \mathbb{Q} / \mathbb{Z})$ of some injective $R$-module $E$. Thus, $\operatorname{Hom}_{R}(P, C)$ is acyclic for every flat cotorsion $R$-module $C$. 
Let $G$ be a flat $R$-module with cotorsion coresolution $G \rightarrow C$. The fact that $\operatorname{Hom}_{R}\left(P, C^{i}\right)$ is acyclic for every $i$ implies by [2, Lemma 2.5] that $\operatorname{Hom}_{R}(P, C)$ is acyclic. Consider the augmented coresolution

$$
G^{+}=0 \longrightarrow G \longrightarrow C^{0} \longrightarrow C^{1} \longrightarrow \cdots \longrightarrow C^{i} \longrightarrow C^{i+1} \longrightarrow \cdots \text {. }
$$

It is a pure acyclic complex of flat $R$-modules, so by [9, Theorem 8.5] the complex $\operatorname{Hom}_{R}\left(P, G^{+}\right)$is acyclic. Since the complex $G^{+}$is the mapping cone of the morphism $G \rightarrow C$, one now has $\mathrm{H}\left(\operatorname{Hom}_{R}(P, G)\right) \cong \mathrm{H}\left(\operatorname{Hom}_{R}(P, C)\right)=0$.

Claim 2.5. Let $R$ be coherent with splf $R$ finite. Every totally acyclic complex $P$ of projective $R$-modules is F-totally acyclic.

Proof. Let $P$ be a totally acyclic complex of projective $R$-modules and $E$ be an injective $R$-module. The complex $P \otimes_{R} E$ is acyclic if the character complex

$$
\operatorname{Hom}_{\mathbb{Z}}\left(P \otimes_{R} E, \mathbb{Q} / \mathbb{Z}\right) \cong \operatorname{Hom}_{R}\left(P, \operatorname{Hom}_{\mathbb{Z}}(E, \mathbb{Q} / \mathbb{Z})\right)
$$

is acyclic. The $R$-module $\operatorname{Hom}_{\mathbb{Z}}(E, \mathbb{Q} / \mathbb{Z})$ is flat, see [12, Lemma 3.1.4], so by assumption it has finite projective dimension. As $\operatorname{Hom}_{R}(P, Q)$ is acyclic for every projective $R$-module $Q$, it follows that $\operatorname{Hom}_{R}\left(P, \operatorname{Hom}_{\mathbb{Z}}(E, \mathbb{Q} / \mathbb{Z})\right)$ is acyclic.

Claim 2.6. Let $R$ be noetherian. Every acyclic complex of flat $R$-modules is totally acyclic if and only if $R_{\mathfrak{p}}$ is Gorenstein for every prime ideal $\mathfrak{p}$ of $R$.

Proof. "If": Let $F$ be an acyclic complex of flat $R$-modules and $E$ be an injective $R$-module. The complex $F \otimes_{R} E$ is acyclic if $\left(F \otimes_{R} E\right)_{\mathfrak{p}} \cong F_{\mathfrak{p}} \otimes_{R_{\mathfrak{p}}} E_{\mathfrak{p}}$ is acyclic for every prime $\mathfrak{p}$. Fix $\mathfrak{p}$; the complex $F_{\mathfrak{p}}$ of flat $R_{\mathfrak{p}}$-modules is acyclic and, since $R_{\mathfrak{p}}$ is Gorenstein, the injective $R_{\mathfrak{p}}$-module $E_{\mathfrak{p}}$ has finite flat dimension; hence $F_{\mathfrak{p}} \otimes_{R_{\mathfrak{p}}} E_{\mathfrak{p}}$ is acyclic.

"Only if": Fix a prime $\mathfrak{p}$. The local ring $R_{\mathfrak{p}}$ is Gorenstein if its $\mathfrak{p} R_{\mathfrak{p}}$-adic completion $\widehat{R_{\mathfrak{p}}}$ is Gorenstein. As an $R$-module $\widehat{R_{\mathfrak{p}}}$ is flat, so every injective $\widehat{R_{\mathfrak{p}}}$-module is injective over $R$. Let $P$ be an acyclic complex of projective $\widehat{R_{\mathfrak{p}}}$-modules; it is a complex of flat $R$-modules and hence F-totally acyclic. Thus, for every injective $\widehat{R_{\mathfrak{p}}}$-module $E$ the complex $P \otimes_{R} E \cong P \otimes_{\widehat{R_{\mathfrak{p}}}} E$ is acyclic. That is, $P$ is F-totally acyclic and hence totally acyclic by Claim 2.4. Being a complete local ring, $\widehat{R_{\mathfrak{p}}}$ has a dualizing complex, and it follows from [6, Corollary 5.5] that it is Gorenstein.

Remark 2.7. We do not know if condition (2.7.1) below is equivalent to finiteness of splf $R$; it is not even clear that it implies finite projective dimension of the flat $R$-module $\prod_{\mathfrak{p}} R_{\mathfrak{p}}$.

$$
\text { proj. } \operatorname{dim}_{R} R_{\mathfrak{p}}<\infty \text { for every prime ideal } \mathfrak{p} \text { of } R \text {. }
$$

We show in Claim 2.8 that $(4) \Rightarrow(1)$ holds if $R$ is noetherian and satisfies (2.7.1). Thus all four conditions in Theorem 1.1 are equivalent for such rings.

Claim 2.8. Let $R$ be noetherian. If proj. $\operatorname{dim}_{R} R_{\mathfrak{p}}$ is finite for every prime ideal $\mathfrak{p}$ of $R$, then every totally acyclic complex of projective $R$-modules is F-totally acyclic.

Proof. Let $P$ be a totally acyclic complex of projective $R$-modules and let $E$ be an injective $R$-module. The complex $P \otimes_{R} E$ is acyclic if $\left(P \otimes_{R} E\right)_{\mathfrak{p}} \cong P \otimes_{R} E_{\mathfrak{p}}$ is acyclic for every prime $\mathfrak{p}$. Fix $\mathfrak{p}$; it is sufficient to prove that the character complex

$$
\operatorname{Hom}_{\mathbb{Z}}\left(P \otimes_{R} E_{\mathfrak{p}}, \mathbb{Q} / \mathbb{Z}\right) \cong \operatorname{Hom}_{R}\left(P, \operatorname{Hom}_{\mathbb{Z}}\left(E_{\mathfrak{p}}, \mathbb{Q} / \mathbb{Z}\right)\right)
$$


is acyclic. The $R_{\mathfrak{p}}$-module $\operatorname{Hom}_{\mathbb{Z}}\left(E_{\mathfrak{p}}, \mathbb{Q} / \mathbb{Z}\right)$ is flat, so it has finite projective dimension over $R_{\mathfrak{p}}$. Every projective $R_{\mathfrak{p}}$-module has finite projective dimension over $R$ by the assumption proj. $\operatorname{dim}_{R} R_{\mathfrak{p}}<\infty$. It follows that proj. $\operatorname{dim}_{R} \operatorname{Hom}_{\mathbb{Z}}\left(E_{\mathfrak{p}}, \mathbb{Q} / \mathbb{Z}\right)$ is finite. By assumption $\operatorname{Hom}_{R}(P, Q)$ is acyclic for every projective $R$-module $Q$, and it follows that $\operatorname{Hom}_{R}\left(P, \operatorname{Hom}_{\mathbb{Z}}(E, \mathbb{Q} / \mathbb{Z})\right)$ is acyclic.

\section{REFERENCES}

1. Ladislav Bican, Robert El Bashir, and Edgar E. Enochs, All modules have flat covers, Bull. London Math. Soc. 33 (2001), no. 4, 385-390. MR1832549

2. Lars Winther Christensen, Anders Frankild, and Henrik Holm, On Gorenstein projective, injective and flat dimensions - A functorial description with applications, J. Algebra 302 (2006), no. 1, 231-279. MR2236602

3. Sergio Estrada, Xianhui $\mathrm{Fu}$, and Alina Iacob, Totally acyclic complexes, Preprint arXiv:1603.03850v2 [math.AC]

4. Laurent Gruson and Christian U. Jensen, Dimensions cohomologiques reliées aux foncteurs $\lim ^{(i)}$, Paul Dubreil and Marie-Paule Malliavin Algebra Seminar, 33rd Year (Paris, 1980),

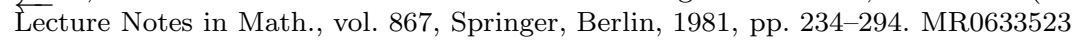

5. Robin Hartshorne, Residues and duality, Lecture notes of a seminar on the work of A. Grothendieck, given at Harvard 1963/64. With an appendix by P. Deligne. Lecture Notes in Mathematics, vol. 20, Springer-Verlag, Berlin, 1966. MR0222093

6. Srikanth Iyengar and Henning Krause, Acyclicity versus total acyclicity for complexes over Noetherian rings, Doc. Math. 11 (2006), 207-240. MR2262932

7. Christian U. Jensen, On the vanishing of $\lim ^{(i)}$, J. Algebra 15 (1970), 151-166. MR0260839

8. Daniel Murfet and Shokrollah Salarian, Totally acyclic complexes over Noetherian schemes, Adv. Math. 226 (2011), no. 2, 1096-1133. MR2737778

9. Amnon Neeman, The homotopy category of flat modules, and Grothendieck duality, Invent. Math. 174 (2008), no. 2, 255-308. MR2439608

10. Michel Raynaud and Laurent Gruson, Critères de platitude et de projectivité. Techniques de "platification" d'un module, Invent. Math. 13 (1971), 1-89. MR0308104

11. Jan Štovíček, On purity and applications to coderived and singularity categories, preprint, arXiv:1412.1615v1 [math.CT]

12. Jinzhong $\mathrm{Xu}$, Flat covers of modules, Lecture Notes in Mathematics, vol. 1634, SpringerVerlag, Berlin, 1996. MR1438789

L.W.C. Texas Tech University, LubBock, TX 79409, U.S.A.

E-mail address: lars.w.christensen@ttu.edu

URL: http://www. math.ttu.edu/ ${ }^{\sim}$ lchriste

K.K. Osaka Prefecture University, Sakai, Osaka 599-8531, Japan

E-mail address: kiriko@mi.s.osakafu-y.ac.jp

$U R L:$ http://www.mi.s.osakafu-u.ac.jp/ kiriko 